\title{
An atom optics experiment to investigate faster-than-light tunneling
}

\author{
A.M. Steinberg, S. Myrskog, Han Seb Moon*, Hyun Ah Kim*, Jalani Fox, and Jung Bog Kim* \\ Department of Physics, University of Toronto \\ Toronto, ONT M5S 1A7 CANADA \\ * Permanent address: Dept. of Physics, Korea Nat. Univ. of Education, \\ Chungbuk, Korea 363-791
}

(March 23, 2021)

\begin{abstract}
We describe a series of atom optics experiments underway at Toronto for investigating tunnelling interaction times of various sorts. We begin by discussing some outstanding issues and confusions related to the question of whether or not superluminal tunnelling can be construed as true fasterthan-light "signal propagation," a question which we answer in the negative. We then argue that atom optics is an arena ideally suited for addressing a variety of remaining questions about how, where, and for how long a particle interacts with a tunnel barrier. We present recent results on a modified "delta-kick cooling" scheme which we have used to prepare Rubidium atoms with onedimensional de Broglie wavelengths on the order of an optical wavelength, along with simulations showing that from these temperatures, we will be able to use acousto-optically modulated dipoleforce barriers to velocity-select ultracold atom samples ideal for future tunnelling experiments.
\end{abstract}

\section{INTRODUCTION: SUPERLUMINAL TUNNELLING AND INFORMATION}

How long does it take a particle to tunnel through a barrier? This question, first raised by 1932 [1] [3], still arouses extreme controversy today (see, e.g., [4,5]). The subject went through a renaissance theoretically in the 1980s [6 8], followed by much experimental work in the 1990s, particularly using electromagnetic analogs to tunnelling such as single-photon transmission through "photonic bandgap" media [9] and microwave transmission through waveguides beyond cutoff [10]. We will not review our single-photon experiments here, as they have been extensively discussed elsewhere. In particular, we refer the reader to a recent review [11], in which we discuss the history of the tunnellingtime conundrum, along with the experiments on electromagnetism and the thorny issues of superluminality and causality.

For what the experiments have confirmed is the validity of the stationary-phase approximation for wave packet propagation through a tunnel barrier (a time frequently referred to as the "phase time," but which we prefer to term the "group delay" in order to avoid misunderstandings about phase vs. group velocities). At the heart of the problem is the fact that the kinetic energy of a particle inside a tunnel barrier is negative, so that a semiclassical estimate of its velocity becomes imaginary. This makes it impossible to make the naïve first approximation that the duration of a tunnelling event is the barrier width divided by the velocity $\sqrt{2 E / m}$. Furthermore, it means that the wave vector inside the forbidden region is imaginary, leading to exponential decay (or growth), in lieu of the accumulation of phase. As no phase is accumulated (except near the boundaries, where the relative amplitudes of the evanescent and anti-evanescent waves are varying significantly), the group delay becomes independent of barrier thickness for "opaque" barriers (i.e., barriers much wider than an exponential decay length). For wide enough barriers, a wave packet peak may emerge from the far side at a time significantly shorter than what would be required for a peak travelling at the vacuum velocity of light $c$. As long as the bandwidth of the signal (typically taken to be gaussian in theoretical work, as well as in certain experiments) is sufficiently small, an essentially undistorted (but greatly attenuated) pulse appears to have travelled faster than light.

As explained in [11 and references therein, no information can be sent faster than light using these effects. To sum the arguments up briefly, the wave exiting the barrier can be expressed by integrating a perfectly causal response function:

$$
\Psi(x=d, t)=\int_{0}^{\infty} d \tau f(\tau) \Psi(x=0, t-d / c-\tau) .
$$

The behaviour of $\Psi(x=0)$ at times $t_{0}>t-d / c$ has no impact whatever on the behaviour of $\Psi(x=d, t)$. Remarkably, the effect of a tunnel barrier is to perform an extrapolation into the future (a Taylor expansion [12,13]) by combining paths with slightly different delays $180^{\circ}$ out of phase with one another [14, 15]. Whether for a gaussian wave packet or a piece by Mozart, the outcome is a convincing reproduction of the incident wave form; some workers therefore argue that signal propagation for such frequency-band-limited waves indeed occurs faster than $c$ [16]. 
Naturally, the difficulty lies with attempts to define (or to avoid defining) "information" and "signal." Only recently have workers attempted to apply a rigorous definition à la Shannon [17], but in our opinion, this application has not yet clarified matters. The cases of superluminal propagation all occur when the "advance" of the transmitted wave remains within a region over which the function can be analytically continued. It is well known that no abrupt disturbance would propagate faster than $c$, and recent electronic-circuit experiments [18] demonstrate that this is entirely consistent with the ability of superluminal systems to "guess" the future by analytic continuation- the new feature is simply that at any point of nonanalyticity, the system will guess wrong.

Those who argue for superluminal signal velocities suggest that since bandwidths are always limited in practice, such abrupt fronts and "points of nonanalyticity" do not exist. A signal defined practically, in terms of a well-defined waveform with a good signal-to-noise ratio, may indeed arrive sooner when tunnelling than when propagating freely (although the time-integrated power will always be less in the presence of the barrier). The question of how best to approximate physical reality - with infinite tails in the frequency domain and abrupt changes in time, or with infinite "precursors" in time and sharp cutoffs in frequency- is an interesting one, often sidestepped. Alas, we choose for now to remain within this tradition. In our opinion, when one talks of a signal velocity, one implicitly refers to the velocity of new information arriving at a receiver. If one accepts that a transmitted waveform is strictly band-limited in frequency, and can therefore be predicted well in advance of its peak by analytic continuation, then the peak does not carry any new information. When information is found at the receiver, there is no reason to refer this back to the time of emission of the peak. In particular, equation (1) shows that information about $x=0$ at times $t<-d / c$ will always suffice to predict what a receiver observes at $x=d$ at times up to $t=0$. If any new information is sent after $t=-d / c$, the receiver will be unaware of it until a time $t>0$. The possibility of a shape appearing at $t<0$ and resembling a shape emitted at $t>-d / c$ in no way alters this. It means merely that the information available (even under free propagation) was already sufficient for predicting the shape which was to come. (In the case of Mozart travelling less than a metre at several times the speed of light, this is another way of saying that it is possible to extrapolate a $10-20 \mathrm{kHz}$ signal a nanosecond into the future without significant distortion!) In sum, a tunnel barrier acts as a marvelous analog computer for signal processing. Under appropriate circumstances, it can take the leading edge of a signal and reshape it into a facsimile of the signal's main content. While in conjunction with amplification 19], this may even turn out to be useful, it is no different from what a digital signal processing chip could do by application of equation (1) to a freely-propagating waveform. Therefore, as in the latter case, we conclude that tunnelling may reduce the delay necessary to process an incoming signal and extract information from it, but it in no way alters the arrival time of the new information itself.

\section{MOTIVATION: INTERACTION TIMES AND NONLOCALITY}

It has long been recognized [6] that the delay of a wave packet peak need not be the unique definition of a tunnelling time (need not, in fact, have any physical significance at all in the view of those authors). Many other times have been proposed to describe the "interaction" of a particle with a tunnel barrier, or the duration of its "sojourn" or "dwell" in the forbidden region. Using the weak-measurement formalism of Aharonov and Vaidman [20,21], we have found that many of these can be unified, and shown to correspond to a general description of von Neumann-style quantum measurements on a subensemble post-selected to contain only transmitted particles [22,23]. The classic thought-experiment of this sort is the Larmor clock [24], but application of the weak-measurement formalism makes it straightforward to obtain physical interpretations of the several components of the Larmor time, as well as their generalisation to a broader class of measurements.

As discussed in 25], a number of these predictions are striking enough to merit experimental test. Summarized briefly, we predict that as defined by the outcome of a classically-conceived measurement, the time spent by a tunnelling particle near the middle of a barrier is exponentially small. On the other hand, transmitted particles spend roughly equal amounts of time near the two classical turning points, while reflected particles only spend time near the entrance. Throughout the entire region, attempts to detect the particle do on the other hand lead to quantum back-action, which is at the origin of the interpretational difficulties.

Perhaps more interesting, it appears that for these post-selected subensembles, there may be a measurable sense in which a particle can be in two places at one time. Here we are not referring to the well-known fact that a wave function passes through both slits of a two-slit interferometer, or in general to the idea that every outcome is an integral over many Feynman paths. In these cases, no attempt is made to measure the position of the particle at the slits. Most discussion centers on how such a measurement, if sufficiently accurate to determine which slit is traversed, destroys interference. We are interested in the reverse scenario. We consider such a gentle, Larmor-style measurement that on no individual occasion can we tell whether or not a particle was observed. By building up enough statistics, 
we can nevertheless draw conclusions about how much time the average particle spent interacting with the measuring device. In the Larmor framework, the particle is a spin initially polarized along the $x$-direction, and the measuring apparatus is a weak magnetic field along $z$, confined to a small region of space. This field sets a Larmor precession frequency $\omega_{L}$, and a particle spending some length of time $t \ll 1 / \omega_{L}$ in the interaction region will precess through an angle $\theta_{y}=\omega_{L} t$. For a spin- $1 / 2$ particle, this angle is of course only measurable by averaging over many trials, but it is straightforward to separate transmitted and reflected particles, so as to measure their polarisations (and hence dwell times) separately.

We have made predictions for the outcome of such measurements, in the form of time-dependent "conditional probability distributions" [23]. These distributions reflect the anomalously fast (potentially superluminal) arrival of a wave packet peak. In fact, for thick enough barriers, one can choose spacelike-separated regions of spacetime centered at opposite edges of the barrier in such a way that the conditional probability of the particle being in either one is arbitrarily close to $100 \%$, since the wave packet peaks themselves are spacelike-separated. The question arises: can a single particle have an effect on two spacelike-separated measuring devices? There is no problem with causality, since the emission of the particle may be in causal contact with both measuring devices. Only the locality of a quantum particle is called into question, but in a way which cannot be directly addressed in familiar situations such as the two-slit experiment, the EPR effect, or the Aharonov-Bohm effect.

Consider a Gedankenexperiment along the lines illustrated in the spacetime diagram of Figure 1. A wave packet tunnels superluminally through a barrier wide enough that the bulk of the transmitted packet is causally disconnected from the bulk of the incident packet. In this case, two weak-measurement devices could be turned on in such a way that the incident particles have a probability arbitrarily close to $100 \%$ of interacting with the first, the transmitted particles have a probability arbitrarily close to $100 \%$ of interacting with the second, and that the two measurement regions are entirely spacelike separated. The two measurement regions might, for example, be pulsed magnetic fields pointing along $+\mathbf{z}$ to the left of the barrier and along $\mathbf{- z}$ to the right of the barrier. In this way, a spin will have precessed in one direction if it sees only the first measurement region, in the other direction if it sees only the second, and not at all if it sees both for equal lengths of time. After transmission, the particles are filtered in energy in such a way that their time of emission can no longer be determined, and it is impossible to trace a particle's trajectory back to either region.

One could conceive of a model of reality which forbids any single particle from being affected measurably by two spacelike-separated devices. Most physicists seem to subscribe to such a model, and it is not yet known whether this is truly consistent with quantum mechanics. Based on the weak measurement picture, we expect that each particle will indeed be affected by both measuring devices. In other words, if only one field or the other is switched on, the spins will precess. But if both fields are on, rather than observing a mixture of spins which have precessed clockwise and spins which have precessed counter-clockwise, we believe that the spins will remain essentially unaffected. This is supported by calculations using the time-independent Schrödinger equation, which show that to first order, the effects of two such Larmor fields cancel out identically. We are currently planning to perform true time-dependent calculations. We intend to construct inequalities constraining "corpuscular" models in which a particle cannot be affected by both fields, and to test these inequalities by studying tunnelling atoms. This will constitute a test of singleparticle nonlocality of a new sort, related to what Aharonov and coworkers term the "reality of the wave function" [26.

\section{THE ATOM OPTICS EXPERIMENT}

We believe that in order to study these quantum subensembles, laser-cooled atoms offer a unique tool. They can routinely be cooled into the quantum regime, where their de Broglie wavelengths are on the order of microns, and their time evolution takes place in the millisecond regime. They can be directly imaged, and if they are made to impinge on a laser-induced tunnel barrier, transmitted and reflected clouds should be spatially resolvable. With various internal degrees of freedom (hyperfine structure as well as Zeeman sublevels), they offer a great deal of flexibility for studying the various interaction times and nonlocality-related issues. In addition, extensions to dissipative interactions and questions related to irreversible measurements and the quantum-classical boundary are easy to envision. [25]

We are working on an atom-optics experiment which will let us directly test these questions. We start with a sample of laser-cooled Rubidium atoms transferred from a magneto-optic trap (MOT) and optical molasses cooling phase to a magnetic trap at approximately $6 \mu \mathrm{K}$. We plan to use a tightly focussed beam of intense light detuned far to the blue of the D2 line to create a dipole-force potential for the atoms [27 29]. Using a $500 \mathrm{~mW}$ laser at $770 \mathrm{~nm}$, we will be able to make repulsive potentials with maxima on the order of the Doppler temperature of the Rubidium vapour. Acousto-optical modulation of the beam will let us shape these potentials with nearly total freedom, such that we 
can have the atoms impinge on a thin plane of repulsive light, whose width would be on the order of the cold atoms' de Broglie wavelength. This is because the beam may be focussed down to a spot several microns across (somewhat larger than the wavelength of atoms in a MOT, but of the order of that of atoms just below the recoil temperature, and hence accessible by a combination of cooling and selection techniques). This focus may be rapidly displaced [30,31 by using acousto-optic modulators. As the atomic motion is in the $\mathrm{mm} / \mathrm{sec}$ range, the atoms respond only to the time-averaged intensity, which can be arranged to have a nearly arbitrary profile.

As a second stage of cooling, we follow the MOT and optical molasses with an improved variant of a proposal termed "delta-kick cooling" [32]. In our version, the millimetre-sized cloud is allowed to expand for about ten milliseconds, to several times its initial size. This allows individual atoms' positions $x_{i}$ to become strongly correlated with atomic velocity, $x_{i} \approx v_{i} t_{\text {free. }}$ Magnetic field coils are then used in either a quadrupole or a harmonic configuration to provide a position-dependent restoring force for a short period of time. By proper choice of this impulse, one can greatly reduce the rms velocity of the atoms. Figure 2 shows free-expansion data comparing atoms expanding from an optical molasses with and without a quadrupole delta-kick. As can be seen by the narrow horizontal width of the latter distribution at late times (the narrow peak is difficult to distinguish from these pictures at early times, but already present in the raw data), the thermal velocity has been greatly reduced. The one-dimensional temperature appears to be about $700 \mathrm{nK}$, corresponding to a de Broglie wavelength of about half a micron. We are currently working on improving this temperature by producing stronger, more harmonic potentials, and simultaneously providing an antigravity potential in order to increase the interaction time.

However, the tunnelling probability through a 5-micron focus will still be negligible at these temperatures. Furthermore, the exponential dependence of the tunnelling rate on barrier height will be difficult to distinguish from the exponential tail of a thermal distribution at high energies. We will therefore follow the delta-kick with a velocityselection phase [25]. By using the same beam which is to form a tunnel barrier, but increasing the width to many microns, we will be able to "sweep" the lowest-energy atoms from the center of the magnetic trap off to the side, leaving the hotter atoms behind. Figure 3 shows the results of simulations for atoms at an initial temperature of $900 \mathrm{nK}$, trapped in a $5 \mathrm{G} / \mathrm{cm}$ field (corresponding to $300 \mu \mathrm{K} / \mathrm{cm}$ ). Superposing a 20- $\mu \mathrm{m}$ gaussian beam with a peak height of $300 \mathrm{nK}$ onto this V-shaped potential creates a local minimum with a depth of $70 \mathrm{nK}$, supporting a single quasi-bound state with a kinetic energy of $13 \mathrm{nK}$. Translating this barrier through the cloud at $0.5 \mathrm{~mm} / \mathrm{s}$ is seen to transfer about $7 \%$ of the atoms into this ground state. This new, smaller sample will have a thermal de Broglie wavelength of approximately $3.5 \mu \mathrm{m}$, leading to a significant tunnelling probability through a 10-micron barrier. We expect rates on the order of $1 \%$ per secular period, causing the auxiliary trap to decay via tunnelling on a timescale of the order of $100 \mathrm{~ms}$.

Once these cooling and selection techniques are perfected, we will have a unique system in which to study tunnelling. By using optical pumping, stimulated Raman transitions, and other such probes, we will be able to go beyond simple wave packet studies to investigate the interactions of tunnelling atoms while in the forbidden region itself. In this way, we hope to shed new light on this fascinating phenomenon, but also on nonlocality in quantum mechanics more generally.

[1] E. U. Condon, Rev. Mod. Phys. 3, 43 (1931); see pp $74 \mathrm{ff}$.

[2] L. A. MacColl, Phys. Rev. 40, 621 (1932).

[3] E. P. Wigner, Phys. Rev. 98, 145 (1955).

[4] G. Nimtz, A. Enders, and H. Spieker, J. Phys. I France 4, 565 (1994).

[5] A. M. Steinberg, J. Phys I France 4, 1813 (1994).

[6] M. Büttiker and R. Landauer, Phys. Rev. Lett. 49, 1739 (1982).

[7] E. H. Hauge and J. A. Støvneng, Rev. Mod. Phys. 61, 917 (1989).

[8] R. Landauer and Th. Martin, Rev. Mod. Phys. 66, 217 (1994).

[9] A. M. Steinberg, P. G. Kwiat, and R. Y. Chiao, Phys. Rev. Lett. 71, 708 (1993).

[10] A. Enders and G. Nimtz, J. Phys. I France 3, 1089 (1993).

[11] R.Y. Chiao and A.M. Steinberg, Progress in Optics vol. XXXVII, pp 345-405, edited by E. Wolf, Elsevier (Amsterdam: 1997).

[12] Y. Japha and G. Kurizki, Phys. Rev. A 53, 586 (1996).

[13] G. Diener, Phys. Lett. A 223, 327 (1996).

[14] A. M. Steinberg, P. G. Kwiat, and R. Y. Chiao (1993). In "Perspectives in Neutrinos, Atomic Physics and Gravitation, 
XXVIIIe Rencontre de Moriond," J. Trân Thanh Vân et al. (eds.), Gif-sur-Yvette, France, Editions Frontières.

[15] A. M. Steinberg, "Effet tunnel: Plus vite que la lumière?", La Recherche 281, 46 (November, 1995).

[16] G. Nimtz and W. Heitmann, Prog. Quant. Electr. 21, 81 (1997).

[17] G. Kurizki, A. Kozhekin, and A.G. Kofman, Europhys. Lett. 42, 499 (1998).

[18] M. W. Mitchell and R. Y. Chiao, Phys. Lett. A 230, 133 (1997).

[19] A. M. Steinberg and R. Y. Chiao, Phys. Rev. A 49, 2071 (1994).

[20] Y. Aharonov, D. Z. Albert, and L. Vaidman, Phys. Rev. Lett. 60, 1351 (1988).

[21] Y. Aharonov and L. Vaidman, Phys. Rev. A41, 11 (1990).

[22] A. M. Steinberg, Phys. Rev. Lett. 74, 2405 (1995).

[23] A. M. Steinberg, Phys. Rev. A 52, 32 (1995).

[24] M. Büttiker, Phys. Rev. B27, 6178 (1983).

[25] A.M. Steinberg, Superlatt. and Microstruct. 23, 823 (1998).

[26] Y. Aharonov, J. Anandan and L. Vaidman, Phys. Rev. A 47, 4616 (1993).

[27] S. L. Rolston, C. Gerz, K. Helmerson, P. S. Jessen, P. D. Lett, W.D. Phillips, R.J.C. Spreeuw, and C. I. Westbrook, "Trapping atoms with optical potentials," in SPIE vol. 1726 Shanghai International Symposium on Quantum Optics, Wang, Wang, and Wang eds., p. 205 (1992).

[28] J.D. Miller, R.A. Cline and D.J. Heinzen, Phys. Rev. A 47, R4567 (1993).

[29] N. Davidson, Heun Jin Lee, C. S. Adams, M. Kasevich, and S. Chu, Phys. Rev. Lett. 74, 1311 (1995).

[30] A. Steinberg, R. Thompson, V. Bagnoud, K. Helmerson, and W. Phillips, In "15th International Conference on Atomic Physics," abstract booklet.

[31] P. Rudy, R. Ejnisman, A. Rahman, S. Lett, and N. P. Bigelow, "Characterization of the RODiO trap," 1997 QELS technical notes, abstract QTuJ5 (1997).

[32] H. Ammann and N. Christensen, Phys. Rev. Lett. 78, 2088 (1997). 


\section{Figure Captions}

Fig. 1. As explained in the text, this Gedankenexperiment is intended to examine whether a single tunnelling particle can be simultaneously affected by two spacelike-separated "weak-measurement devices," for example, pulsed magnetic fields. The superluminally transmitted particle is passed through an energy-filter which erases all information about its time of emission, and the precession angle of its spin should provide information about which of the interaction regions it traversed.

Fig. 2. These fluorescence images show the expansion of a cloud of ultracold Rubidium atoms over a $27 \mathrm{~ms}$ period. The isotropic expansion in the upper sequence, directly from an optical molasses, indicates a temperature of approximately $9 \mu \mathrm{K}$. The lower sequence shows an analogous expansion after a "quadrupole kick" is used to further cool the atoms one-dimensionally. The dark central stripe visible in the final frames has been analyzed, and corresponds to a temperature of approximately $700 \mathrm{nK}$.

Fig. 3. This quantum-mechanical simulation demonstrates what we expect to achieve with our dipole-force velocityselection. Starting with an atom cloud near $1 \mu \mathrm{K}$ and sweeping an appropriately tuned $20 \mu \mathrm{m}$ laser beam through the atoms adiabatically, we will create a very small auxiliary potential well. The probability of transfer exhibits steps as a function of well depth, indicating the number of quasi-bound states supported. A well with a single bound state is seen to capture about $7 \%$ of the atoms, in a state with a kinetic temperature of only $13 \mathrm{nK}$, corresponding to a de Broglie wavelength of about $3.5 \mu \mathrm{m}$. Such a state will be an ideal source for our atom-tunnelling experiments. 


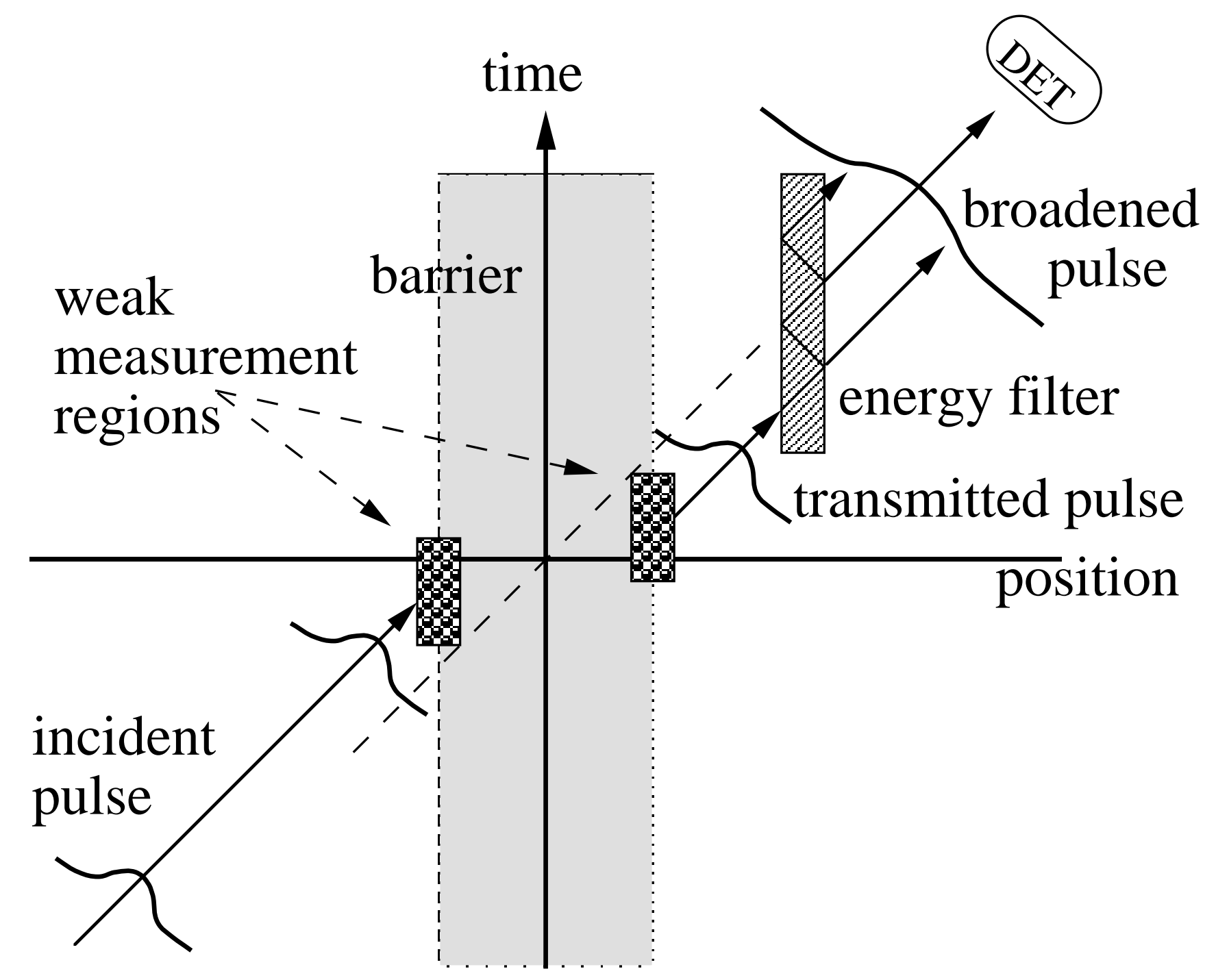




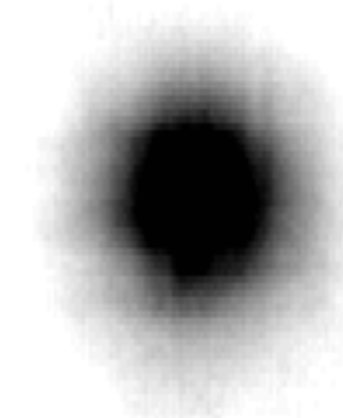

Free Expansion

$15 \mathrm{~ms}$

$18 \mathrm{~ms}$

Time out of Molasses

$\approx 10 \mathrm{~m} / \mathrm{s}^{2}$ kick starts at $9 \mathrm{~ms}$, ends at $12 \mathrm{~ms}$

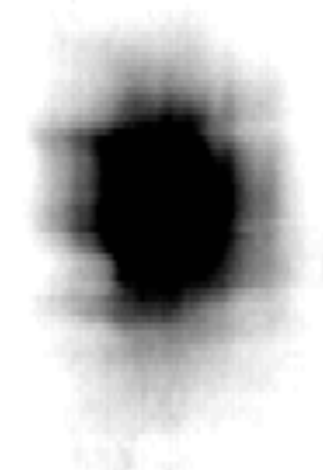

$21 \mathrm{~ms}$

$24 \mathrm{~ms}$

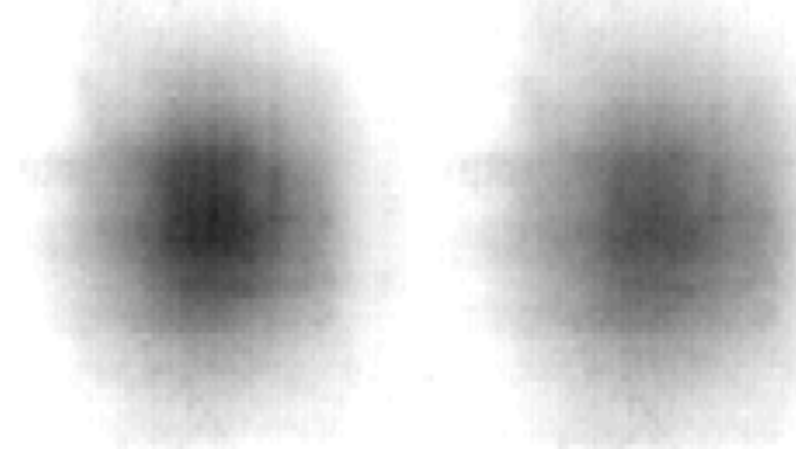

$$
\left(T_{0} \approx 9 \mu \mathrm{K}\right)
$$

$27 \mathrm{~ms}$

Apparent temperature of central feature:

$700 \mathrm{nK}(1.5 \mathrm{Vr})$

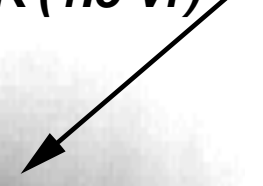

\section{Quadrupole Kick from from a Molasses}




\section{Velocity Selection vs Barrier Height Simulation}

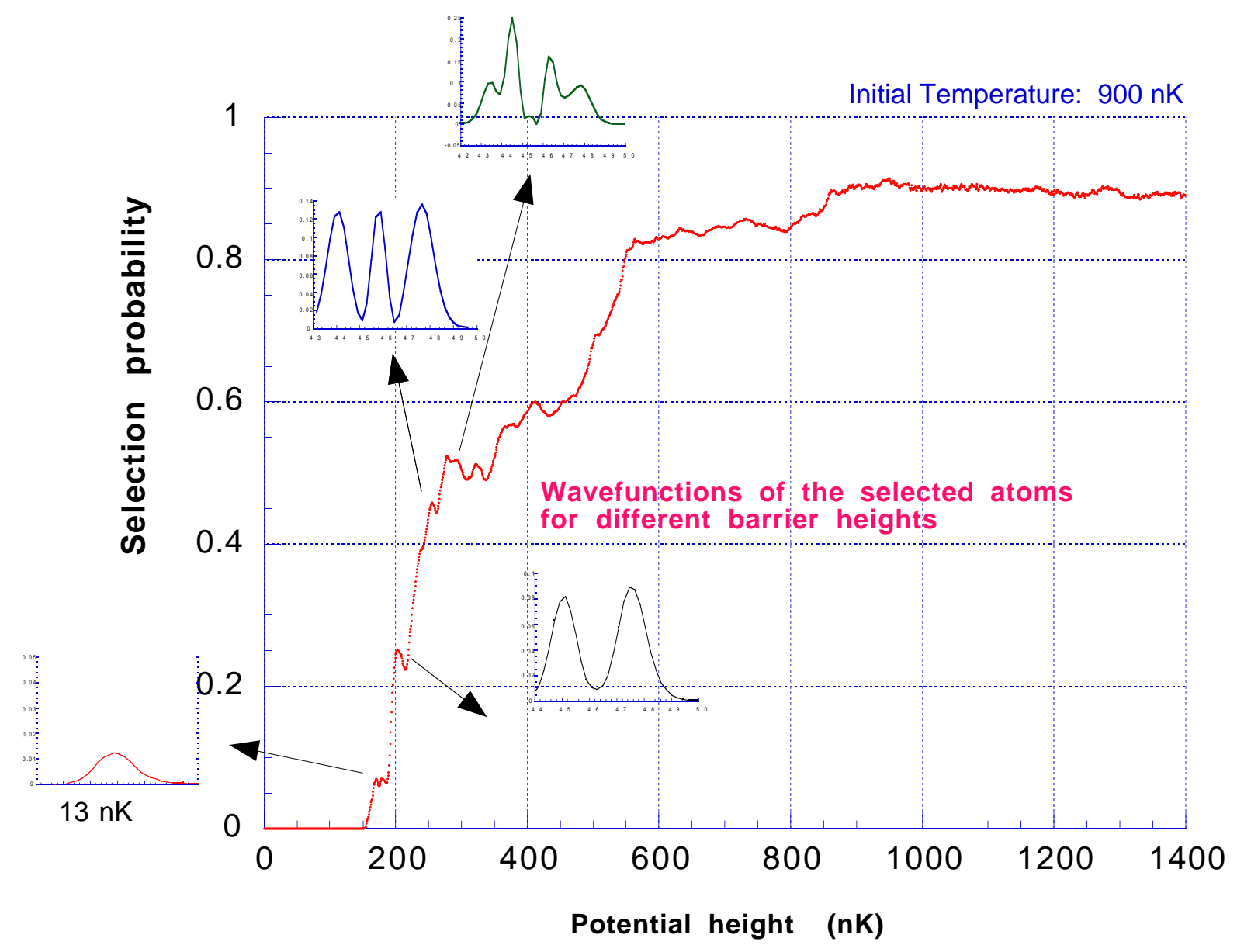

